\title{
Trois thèses de prédécouverte de l'Amérique du Sud par le Pacifique*
}

Par Paul Gallez

\section{Le réseau hydrographique sudaméricain sur une carte précolombine}

Dans une étude publiée récemment, nous avons eu l'occasion de montrer que le réseau hydrographique d'Amérique du Sud était fidèlement représenté sur le ptolémée qui occupe les folios $68 \mathrm{v}^{\circ}$ et $69 \mathrm{r}^{\circ}$ du manuscrit de l'Insularium Illustratum Henrici Martelli Germani qui se trouve au Musée Britannique. Fleuve par fleuve, le dessin correspond à la réalité ${ }^{1}$.

Ceci ne serait que naturel, si ce n'était que le ptolémée a été dessiné en 1489 , trois ans avant le premier voyage de Christophe Colomb, trente ans avant la découverte sommaire des côtes sudaméricaines des deux océans, et quatre siècles avant l'exploration de l'intérieur du continent, principalement de l'Amazonie et de la Patagonie.

La correction des informations dont a disposé le cartographe Hammer (Martellus) qui travailla à Florence de 1480 à 1496, remet sur le tapis le problème des prédécouvertes de l'Amérique du Sud.

Comme ce continent, sur la carte de Hammer, occupe la position extrême de l'Orient, il nous semble évident que les mystérieux explorateurs y sont arrivés par le Pacifique.

Qui étaient-ils? Quand et comment ont-ils fait le voyage? Comment leurs informations cont-elles arrivées aux mains du cartographe allemand de Florence?

Nous n'avons certes pas la prétention de donner à ces questions une réponse définitive. Nous esquisserons les principales thèses de prédécouverte et proposerons quelques idées nouvelles.

Notons qu'il s'agit de thèses non historiques, mais protohistoriques, échafaudées sur la faible base d'indice parfois douteux, et qu'il serait vain et abusif d'en exiger des preuves rigoureuses.

* Cette étude a été réalisée avec l'aide du Conseil National des Recherches Scientifiques et Techniques de la République Argentine. - Nous remercions de ses conseils et de ses critiques M. le Professeur Dr. Hanno Beck, de l'Université de Bonn. 


\section{La thèse égyptienne}

La thèse qui se rapporte aux expéditions les plus anciennes est celle des voyages à la Terre de Punt.

Le premier voyage dont on ait connaissance fut organisé sous le règne du pharaon Sahure de la $\mathrm{V}^{\mathrm{e}}$ dynastie (2550 a. J.C.). Ses marins rapportèrent du Punt et des escales de l'encens, de la myrrhe, de l'or, de l'argent, des bois précieux et des esclaves.

Vers l'an 2400, le pharaon Asa (Isesi) imita son exemple.

Une princesse de la $\mathrm{VI}^{\mathrm{e}}$ dynastie emportait dans sa tombe, pour le voyage vers le pays des morts, du rouge à lèvres à base d'antimoine, métal inconnu à l'époque dans les régions voisines de l'Egypte. Quelques années plus tard mourait Knemhotep, pilote d'Eléphantine, dont la table funéraire indique qu'il a fait onze fois le voyage à la Terre de Punt ${ }^{2}$. Ceci se passait vers l'an 2300 avant notre ère.

D'autres voyages furent organisés par Seanjkaré de la $\mathrm{IX}^{\mathrm{e}}$ dynastie, et plus tard par la reine Hatsepsut (Hatsheput, Hatschepsut, Hachesupt, Hatshopsitou, Haschepsowe, Hatasu, 1501-1480 a. J.C.), qui a laissé dans le temple de Deir-elBahari le récit des expéditions qu'elle envoyait à la Terre de Punt ${ }^{3}$.

L'expédition d'Hatsepsut comportait cinq grands navires de trente rameurs,

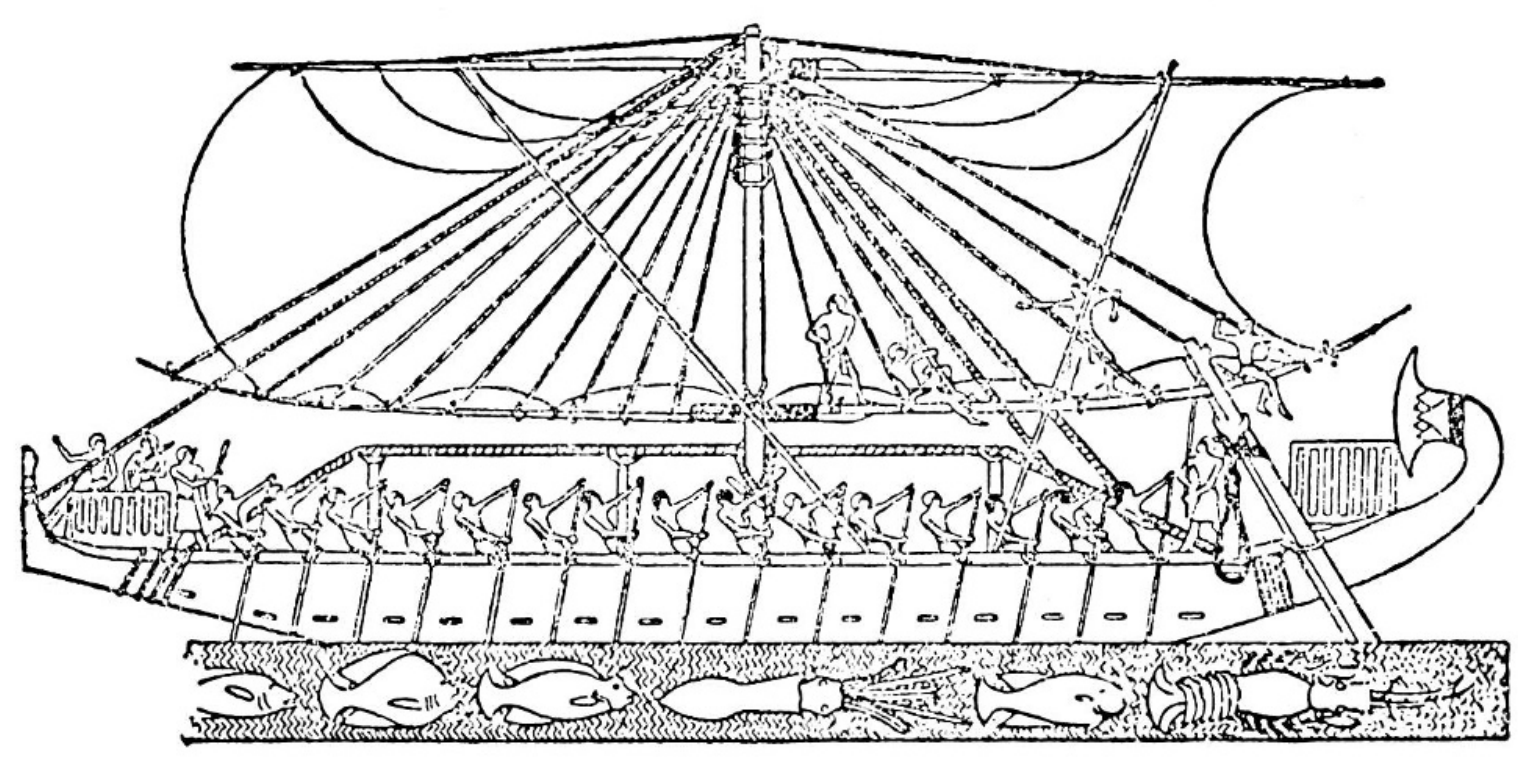

Fig. 1. Bateau expéditionnaire de la reine Hatsepsut ( $\mathrm{XV}^{\mathrm{e}}$ siècle a. J. C.). Gravure du temple de Deir-el-Bahari. Tiré de Paul Herrmann, Sieben vorbei und acht verweht. Das Abenteuer der frühen Entdeckungen. Hamburg 1952, p. 73 
qui partirent par la Mer Rouge en 1493. Le voyage d'aller-et-retour dura trois ans. On en rapporta, comme avant, de l'or, des esclaves, de l'antimoine, des pierres précieuses, etc.

En 1180, le pharaon Ramsés III envoya au Punt une expédition de 10.000 hommes, d'après la relation de Ramsés IV dans le Papyrus Harris.

Les navires égyptiens de haut bord avaient une longueur d'une trentaine de mètres et atteignaient les 80 ou 85 tonnes, c'est-à-dire le même tonnage que les caravelles de Christophe Colomb. Ils avaient le fond plat et pouvaient, par vent favorable, filer à une vitesse considérable. Par temps calme, les rameurs entraient en action et évitaient de devoir attendre le changement de vent.

Plusieurs thèses ont été émises au sujet de la localisation géographique de la Terre de Punt: L'Erythrée, la Somalie, Zimbabwe en Rhodésie, l'Hadramaut et l'Inde.

Nous voudrions suggérer une nouvelle thèse: celle de l'Amérique du Sud, et plus particulièrement la région péruvienne de Puno (Punt?) sur les bords du lac de Titicaca, d'où l'on tire actuellement $70 \%$ de toute la production d'or du Pérou, ainsi que de l'antimoine, du mercure, du zinc, de l'étain et du cobalt. On y trouve de vieilles mines d'or et d'antimoine exploitées à une époque très reculée que l'on n'a pas pu préciser.

Curieuse coïncidence, une colline qui porte le nom de Huansopata (Hatseput?) y est le siège de nombreuses légendes qualifiées de fabuleuses, d'origine aussi lointaine que mystérieuse. Les sépulcres et les ruines de cette colline appartiennent à la civilisation de Tiahuanaco, objet d'opinions aussi savantes que contradictoires.

Les barques du lac Titicaca sont faites de roseaux et ressemblent tellement à celles de l'Ancienne Egypte, que c'est à Puno que Thor Heyerdahl a recruté les hommes qui ont construit, sur les rives du Nil, son bateau de papyrus Ra II. Puno ne se trouve, en ligne droite, qu'à $240 \mathrm{~km}$ de l'Océan Pacifique; les ruines des vallées péruviennes situées entre le lac Titicaca et la côte appartiennent également à la culture de Tiahuanaco ${ }^{4}$.

La thèse de Puno, qu'il faudrait développer, nous semble à première vue aussi valable que les autres. Elle vient d'être renforcée par la découverte réalisée par le Dr. Barry Fell de l'université de Harvard. Dans la vallée chilienne de Tinguiririca, à $34^{\circ} 45^{\prime} \mathrm{S}$ et $70^{\circ} 20^{\prime} \mathrm{W}$, à $150 \mathrm{~km}$ de la côte du Pacifique, Carl Stolp avait relevé en 1889 une inscription que Fell fait remonter à 231 a. J.C. Selon la traduction de Fell, les égyptiens y réclament pour leur roi 4000 milles de côte escarpée qui terminent à la hauteur de Tinguiririca, et commencent done aux environs de l'isthme de Panama. 


\section{La thèse phénicienne}

Les phéniciens font leur entrée dans l'histoire maritime vers l'an 1000 avant notre ère. Cinquante ans plus tard, ils ont acquis un tel prestige nautique, que Salomon demande au roi Hiram de Tyr de lui envoyer des charpentiers pour construire une flotte, ainsi que des marins pour la conduire ${ }^{5}$ vers la pays d'Ophir. Ophir était situé, comme la Terre de Punt, dans le Grand Sud-est, et le voyage aller-et-retour durait également trois ans.

Salomon était gendre du pharaon, et il a probablement reçu des égyptiens les indications nécessaires pour réaliser le voyage au Punt, que les hébreux appelleront Ophir. En effet, les produits rapportés d'Ophir ${ }^{6}$ et des escales sont les mêmes que ceux qui venaient du Punt et des escales. Les problèmes du Punt et ceux d'Ophir sont les mêmes, et il n'y a pas de raison majeure de leur proposer des solutions différentes.

Le Pérou a été proposé souvent sur la base erronée de l'identité des consonnes $\mathbf{P}$ et $\mathbf{R}$ dans $O$ phir $=$ Pérou $^{7}$ alors que le Pérou ne porte ce nom que depuis la conquête espagnole.

Une fois la route d'Ophir révélée par les égyptiens aux hébreux et par les hébreux aux phéniciens, ceux-ci continuèrent les expéditions pour leur propre compte.

Sur les stèles centrales du temple de Sechin dans la vallée de Casma (côte péruvienne, à $9^{\circ} 30^{\prime} \mathrm{S}$ ), Edouard Habich a identifié deux navires phéniciens ${ }^{8}$. On considère généralement que ces ruines datent de 1000 avant J. C. environ. D'autres monolithes représentent un grand navire océanique et un sextant ${ }^{9}$.

Ce qui est bien plus extraordinaire, ce sont les découvertes de Bernardo da Silva Ramos. Cet auteur, président de l'Institut Géographique de Manaos, a passé plus de vingt ans à parcourir la forêt vierge de l'Amazonie pour y chercher, photographier et copier des inscriptions rupestres. Il en a recueilli plus de deux mille huit cents, reconnaissant la plupart d'entre elles comme phéniciennes, et les autres comme grecques ${ }^{10}$.

D'autres inscriptions, comme celle de Gavea près de Rio de Janeiro, parlent des rois phéniciens du $\mathrm{IX}^{\mathrm{e}}$ siècle avant notre ère. Celle de Parahyba a été sommairement classée comme une supercherie moderne.

Il est notable que les autorités politiques et académiques brésiliennes se refusent souvent à examiner ces inscriptions et les considèrent, d'avance, comme de pures inventions de farceurs de notre époque. Il doit être bien facile de distinguer une inscription qui date de 25 ans d'une autre qui ait 25 siècles d'exis- 
tence. Et quel savant passerait des années de sa vie à parcourir la forêt vierge pour y graver sur les rochers des inscriptions pseudo-phéniciennes?

Le mépris et la négligence officiels semblent avoir pour cause subconsciente la crainte d'enlever aux ancêtres portugais la gloire de la première découverte du Brésil.

Il est hors de doute que les navires phéniciens (les bateaux de Tharsis) et ceux de l'époque gréco-romaine étaient capables de réaliser le voyage transpacifique grâce aux courants marins et aux vents réguliers. Il en était de 6500 tonnes, comme les nefs de Ptolémée IV Philopator (222-205 a. J.C.) ou 4200 tonnes comme le Alexandrie de Hiéron II de Syracuse (270-216 a. J.C.). L'historien Flavius Josèphe (37-100) parle de bateaux qui, outre l'équipage, conduisaient six cents passagers et des marchandises ${ }^{11}$.

Les peintures mochicas du nord du Pérou représentent des navires de commerce dotés d'un pont et chargés de jarres, qui ne diffèrent pas notablement des embarcations maritimes représentées sur les mosaïques de la Méditerranée orientale deux ou trois siècles avant notre ère ${ }^{12}$.

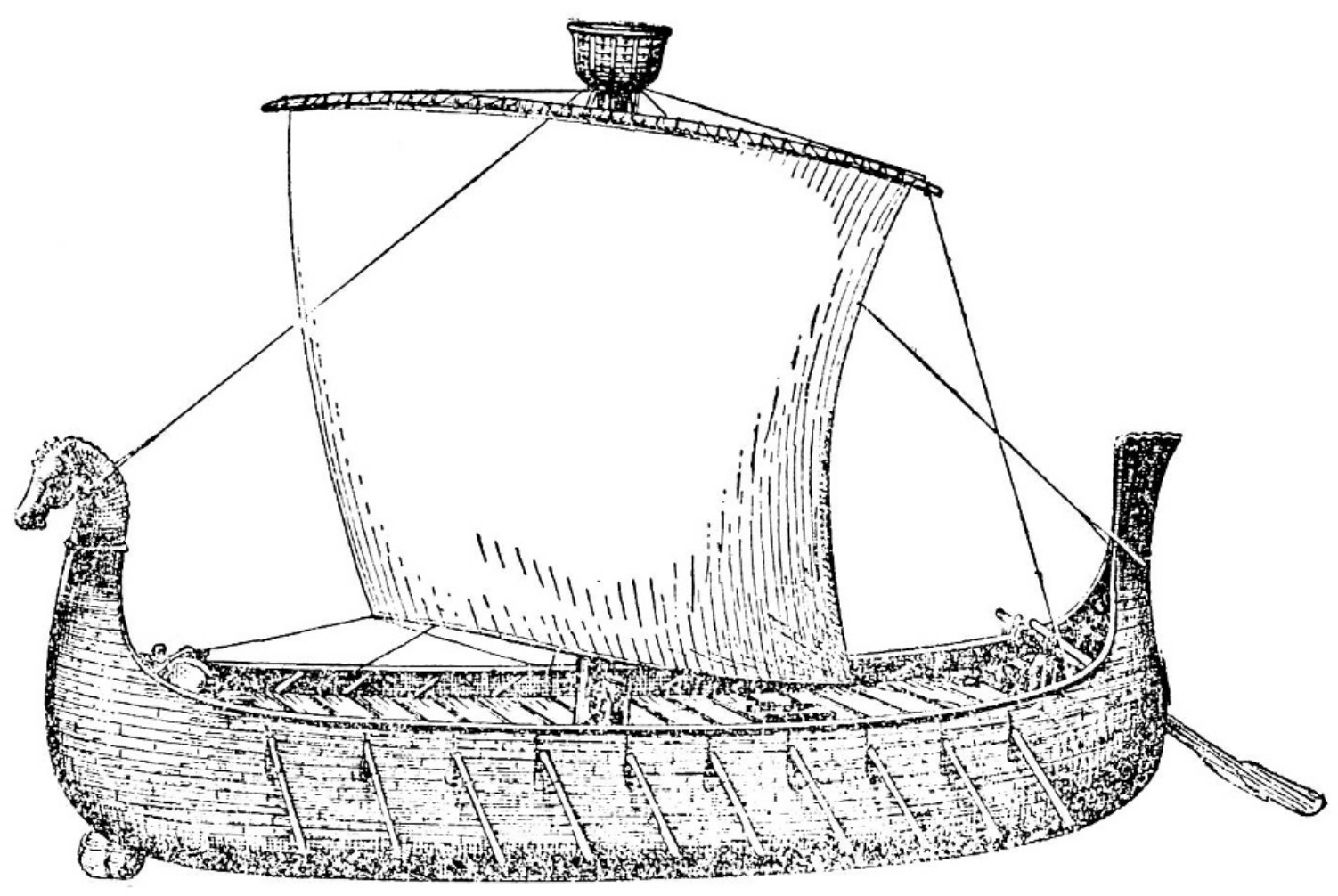

Fig. 2. Bateau phénicien du IX ${ }^{\mathrm{e}}$ siècle a. J. C. Tiré de Paul Herrmann, Sieben vorbei und acht verweht, Hamburg 1952, p. 87 


\section{La thèse chinoise}

La thèse chinoise se divise en trois étapes bien distinctes, au point que l'on pourrait parler de trois thèses chinoises.

La première dérive du Périple de la Mer Erythrêe ${ }^{13}$ et d'un texte de Marin de Tyr qui indique que, dès l'occupation de l'Egypte par les Romains, ceux-ci envoyèrent leurs bateaux marchands par l'Océan Indien vers la Chine et, audelà, en un lointain voyage, à Cattigara.

Certains auteurs ont placé ce port en Chine parce que Ptolémée Je qualifie de Ormos Sinon ${ }^{14}$ ou mouillage des Chinois. Mais il est évident que l'indication «mouillage des Chinois» n'aurait aucune valeur pour un toponyme de Chine, où tous les mouillages sont forcément chinois. C'est donc ailleurs qu'en Chine qu'il faut situer Cattigara. En effet, Ptolémée le situe sur la côte orientale du Sinus Magnus, c'est-à-dire de l'autre côté du Pacifique, sur la côte américaine; il lui donne une latitude de $8^{\circ} 30^{\prime}$ Sud, latitude que le territoire chinois n'a jamais atteinte sur aucune carte.

Ibarra Grasso a identifié Cattigara avec Lambayeque ${ }^{15}$ sur la côte péruvienne, à $6^{\circ} 40^{\prime} \mathrm{S}$. C'est donc le mouillage que les chinois utilisaient sur la côte américaine. En d'autres termes, Ptolémée nous annonce que les Chinois fréquentaient la côte péruvienne et y avaient leur Ormos ou Statio Navium, d'où nous pouvons déduire qu'ils y exerçaient habituellement le commerce.

Le texte de Marin de Tyr indique très clairement que la route maritime passe d'abord par Zaba, situé au-delà de la Chersonèse d'Or, c'est-à-dire de la presqu'île de Malaca. Ibarra Grasso identifie Zaba avec le Sabah actuel, dans le nord de l'île de Bornéo ${ }^{16}$. Permettons nous de suggérer que ce Zaba-Sabah était peutêtre le pays de la reine de Saba, producteur d'or, de bois de santal et de pierres précieuses ${ }^{17}$.

A partir de Zaba, dit Marin de Tyr, il faut mettre le cap «au sud, puis à l'est, et voyager un nombre de jours tellement grand qu'il est impossible de les compter» afin d'arriver à Cattigara, sans escale.

La carte des courants maritimes indique clairement que, de Bornéo vers le sud, le sud-est et l'est, on prend la route du grand courant circulaire dont la branche sud-pacifique va d'ouest en est. Aux approches de l'Amérique, le même courant prend le nom de Humboldt et remonte vers le nord le long des côtes chilienne et péruvienne. Portés par les courants marins et les vents dominants, les égyptiens, les phéniciens et sans doute aussi les chinois (vu le nom de Ormos Sinon) arrivaient ainsi à Cattigara-Lambayeque.

Le retour se faisait en reprenant le même courant circulaire du Pacifique sud, 
qui ramenait les bateaux vers l'ouest et les Moluques, cette fois sous un climat plus clément qu'à l'aller.

La deuxième thèse chinoise se rapporte au III $^{e}$ siècle avant notre ère. C'est la légende de Fou-Sang, pays merveilleux situé très loin à l'est de la Chine, au-delà des mers. Les traditions chinoises en racontent des histoires pleines de mystère. L'empereur Shih-Huang-Ti, en 219 a. J.C., y a envoyé une expédition composée de filles et garçons qui y restèrent et y vécurent très heureux.

La troisième thèse chinoise est née des Annales de la Chine, au $\mathrm{V}^{\mathrm{e}}$ siècle de notre ère. D'après l'écrivain Ma-Twan-Lin, l'an 499, le prêtre bouddhiste chinois Hwui-Shin revint de Fou-Sang, pays situé à 20.000 lis vers l'orient, dont il décrivit les habitants, les coutumes, les maisons, les arbres, les animaux. Hwui-Shin explique que le Fou-Sang a été converti au bouddhisme par cinq moines de KiPing (Afghanistan) en l'an 458.

Fig. 3. Homme de Fou-Sang trayant un lama. Tiré de Gustave Schlegel, Problèmes géographiques: Les peuples étrangers chez les historiens chinois. I. Fou-Sang Kouo, Leide 1892, p. 27 (Attention du Dr. Ortiz Troncoso)

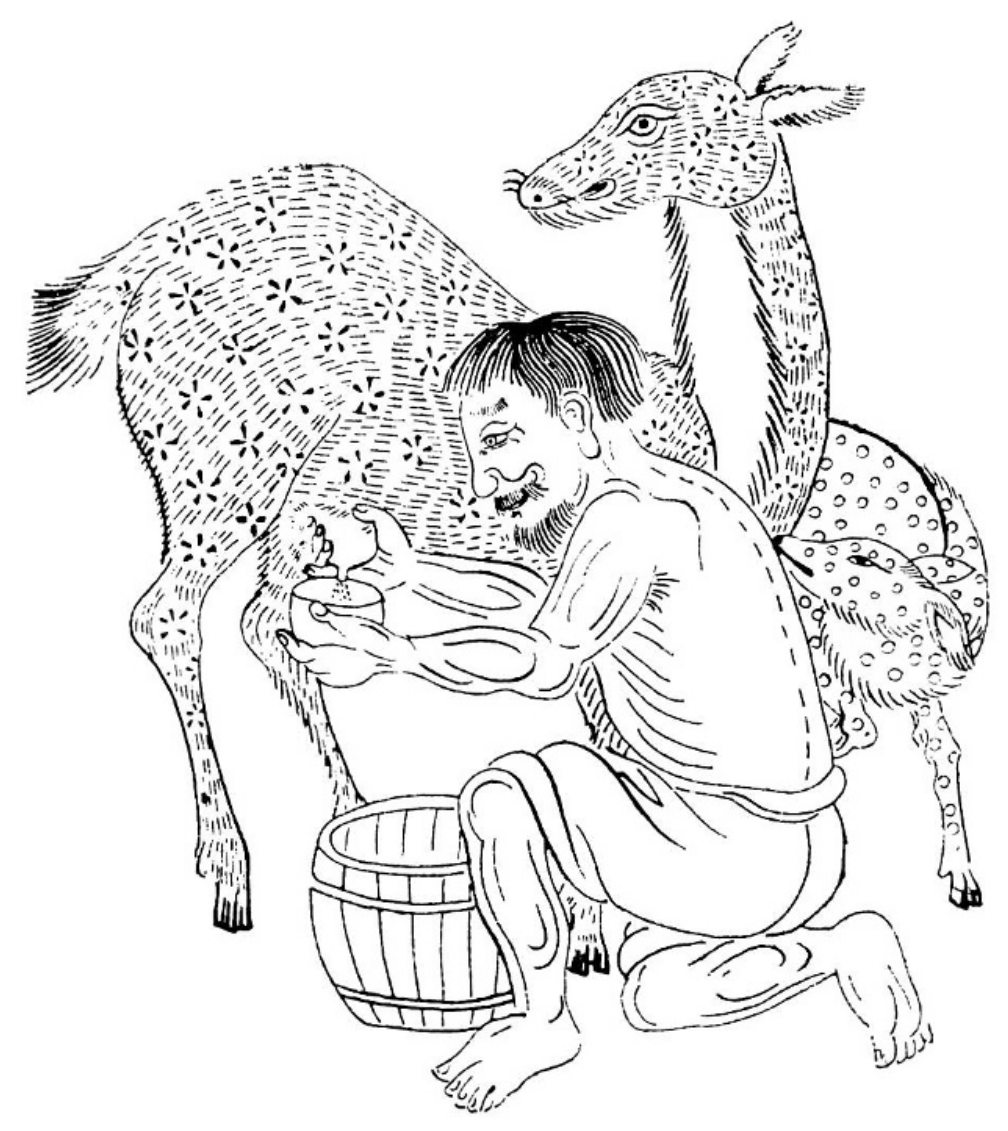


Certains éléments de cette description, comme le dessin d'un homme qui trait un lama, font penser immédiatement au Pérou. Cependant, le premier européen qui ait situé le Fou-Sang en Amérique, le français De Guignes ${ }^{18}$, l'identifiait avec le Mexique, dans une étude qui a fait sensation, en plein $\mathrm{XVIII}^{\mathrm{e}}$ siècle. En 1841, Carl Friedrich Neumann, professeur de langues et d'histoire orientales à l'Université de Munich, a publié une traduction allemande de la partie des Annales qui se rapporte au Fou-Sang. Il situe également cette région en Amérique. La même thèse a été reprise en 1844 par Paravey ${ }^{19}$ et en 1865 par Gustave d'Eichthal. En 1875, Leland traduisit en anglais la traduction allemande de Neumann ${ }^{20}$. L'étude la plus complète est certainement celle de Vinning, publiée en $1885^{21}$, qui défend la thèse de De Guignes avec un grand luxe de détails. Citons encore Hervey de Saint-Denis et, plus récemment, Orti Belmonte ${ }^{22}$.

La thèse de De Guignes a été fortement attaquée en 1831 par Julius Heinrich Klaproth ${ }^{23}$, puis par Vivien de Saint Martin ${ }^{24}$, Bretschneider ${ }^{25}$, Gustave Schlegel $^{26}$, Dall, Müller et Chamberlain ${ }^{27}$. Les uns situent le Fou-Sang au Japon, d'autres à Sakhaline. Une des principales critiques formulées contre les thèses américaines au siècle dernier, était la croyance à l'impossibilité, pour les bateaux chinois du III ${ }^{\mathrm{e}}$ siècle a. J. C. et du $\mathrm{V}^{\mathrm{e}}$ siècle de notre ère, de traverser le Pacifique. Cette opinion a changé aujourd'hui.

\section{Un problème protohistorique à la recherche d'une solution}

Le problème protohistorique peut se diviser en six questions :

1. Prédécouverte: Qui a abordé en Amérique du Sud avant 1428 ou avant 1489 ? Quand et comment?

2. Reconnaissance des côtes: Qui a reconnu les côtes pacifique et atlantique? Quand et comment?

3. Reconnaissance de l'intérieur: Qui a exploré l'intérieur du continent au point de faire un relevé étonnamment correct de son réseau hydrographique? Quand et comment?

4. Centre politique ou culturel: A quelle cour, à quel centre politique ou culturel ces informations ont-elles été transmises? Par qui et quand?

5. Transmission à l'Italie: Qui a retransmis ces informations à Rome ou à une autre ville italienne ou européenne? Quand et comment?

6. Information de Hammer: Qui les a communiquées à Hammer? 
La première question (prédécouverte) obtient trois réponses. Notre thèse de Punt est aussi difficile à prouver ou à réfuter que ses concurrentes de Somalie ou de Rhodésie, mais elle vient d'être renforcée par la découverte de Fell. La thèse phénicienne s'appuie sur les ruines de la vallée de Casma. La chinoise tire ses arguments de Ptolémée et des Annales de Chine. Les courants marins et les vents du Pacifique rendent ces trois thèses possibles. Il est difficile de choisir entre elles.

La deuxième question (reconnaissance des deux côtes océaniques) n'a pas reçu de réponse jusqu'à présent.

La troisième (reconnaissance de l'intérieur) obtient une réponse partielle dont il faudrait vérifier la validité. Les égyptiens, les phéniciens et les chinois étaient probablement des commerçants qui achetaient aux amérindiens les produits locaux. Cependant, les nombreuses inscriptions recueillies par Da Silva Ramos, si elles sont authentiques, indiquent que les égyptiens et les phéniciens auraient réalisé de grandes expéditions terrestres à l'intérieur de l'Amazonie. Le reste du continent n'offre aucun indice de ce genre.

La quatrième question (centre politique ou culturel) dépend des précédentes. Si les pharaons ont connu le secret de la géographie sud-américaine, comment cette science s'est-elle perdue? La réponse est simple: de la même façon que se sont perdus l'itinéraire du Punt (identifié ou non à l'Amérique) et les secrets astronomiques matérialisés dans la Grande Pyramide. Les marchands phéniciens ont perdu leur secret quand ils ont dû abandonner leurs voyages par l'Océan Indien. Les chinois ont transformé le leur en légende quand les guerres internes ont mis fin à leurs grandes randonnées transocéaniques.

La cinquième question (transmission à l'Italie) offre plusieurs possibilités. On peut supposer, sans aucune preuve, que les commerçants arabes ont hérité quelques traditions et légendes égyptiennes ou phéniciennes et les ont transmises $\mathrm{au} \mathrm{XV}^{\mathrm{e}}$ siècle à leurs clients florentins ou vénitiens. Ou que les franciscains qui ont évangélisé la Chine au Moyen-Age y ont appris des secrets qu'ils auraient transmis à Rome.

La sixième et dernière question (information de Hammer) est la plus facile. Hammer travaillait à Florence et cherchait les informations dont il avait besoin pour dessiner ses cartes. Il a pu développer ses relations au Vatican au point de pouvoir y consulter des rapports secrets, ou recevoir des marchands florentins les rapports de leurs représentants à Alexandrie.

Il s'agit d'une série de problèmes enchaînés, qui ne sont pas historiques, car on manque presque totalement de données sûres pour les résoudre. Nous nous voyons donc forcés de les traiter comme des problèmes protohistoriques et à construire de fragiles échafaudages de thèses audacieuses sur la base de faits 
légendaires douteux. Nous savons que n'importe lequel de ces échafaudages peut se renforcer ou s'écrouler à la simple découverte de restes archéologiques insoupçonnés ou à l'apparition d'un document à présent inconnu.

Nous ne pouvons pas exiger de preuves, car il n'y en a pas. Nous pouvons seulement demander que les thèses pleines d'imagination soient possibles et cohérentes.

A notre avis, c'est une erreur de méthode, de vouloir appliquer la critique historique à des thèses protohistoriques. Le seul résultat que l'on peut attendre de ce procédé est la destruction de toutes ces thèses, sans pouvoir les remplacer par aucune autre qui soit capable de résister à ce traitement. Il vaut mieux avoir trois thèses boîteuses et incompatibles que de n'en avoir aucune, car leur propre faiblesse et leurs divergences invitent à raisonner et à perfectionner les idées proposées, à en élaborer d'autres, à approfondir leurs interrelations, et à chercher des voies nouvelles qui souvent s'empalment ou s'entrecroisent avec les précédentes, sauvant celles-ci en partie ou les revalorisant.

Nous avons tenté d'exposer la situation actuelle de ces questions et de définir les problèmes. Nous avons présenté quelques réponses en les appuyant sur des indices, sans pouvoir les étayer d'arguments irréfutables.

La solution dépendra en partie de l'imagination des chercheurs, mais aussi des futures découvertes archéologiques en Amérique et peut-être de recherches dans les archives de Chine et d'Italie.

Notes

1 Paul Gallez, Les grands fleuves d'Amérique du Sud sur le ptolémée londonien d'Henri Hammer. In: Erdkunde XXIX/4, Bonn 1975.

2 Cf. Paul Herrmann, Historia de los descubrimientos geográficos, tome I, Barcelone, Labor, $3^{\mathrm{e}}$ éd. 1968, p. 55-65.

${ }^{3}$ Cf. Dümichen, Die Flotte einer ägyptischen Königin, Leipzig 1868; Naville, Deir el Bahri. In: Mémoires du Egypt Exploration Fund, Londres 1894, et E.Zyhlars, Das Land Punt. In: Zeitschrift für Eingeborenensprachen, Leipzig 1941-42.

${ }^{4}$ Cf. José Imbelloni, La segunda esfinge indiana, Buenos Aires, Hachette, 1956, p. 90 .

5 Ancien Testament, Livre des Rois, I, 9-10.

6 Armand d'Avezac, Mémoire sur le pays d'Ophir où les flottes de Salomon allaient chercher de l'or. In: Mémoires de l'Académie des Inscriptions et BellesLettres XXX, Paris 1864. 
7 Voir la destruction de cette thèse dans José Imbelloni, o. c., p. 38-40.

${ }^{8}$ Cf. Dick Edgar Ibarra Grasso, La representación de América en mapas romanos de tiempos de Cristo, Buenos Aires 1970, p. 176.

9 Julio C.Tello, Arquelogia del Valle de Casma, cité par Ibarra Grasso, o.c., l.c.

10 Bernardo da Silva Ramos, Inscripções et tradições da America prehistorica, Rio de Janeiro 1932.

11 Cf. Herrmann, o.c., p. 159.

12 Cf. Ibarra Grasso, o.c., p. 166.

13 Voir William Vincent, Periplus of the Erythrean Sea, 2 vol., Londres 1800, et W.H.Schoof, Periplous Maris Erythraei, Stuttgart 1912.

${ }^{14}$ Claude Ptolémée, Géographie, Livre VII, chap. III.

15 Ibarra Grasso, o.c., p. 177.

16 Idem, o.c., p. 42.

17 Ancien Testament, Livre des Rois, I, 10.

${ }^{18}$ De Guignes, Le Fou-Sang des chinois est-il l'Amérique? In: Mémoires de l'Académie des Inscriptions et Belles Lettres XXVIII, Paris 1861, p. 505-523.

19 Hippolyte de Paravey, L'Amérique sous le nom de Pays de Fousang est-elle citée dès le cinquième siècle de notre ère dans les Grandes Annales de la Chine et, dès lors, les Samanéens de l'Asie Centrale et du Caboul y ont-ils porté le bouddhisme? Paris, Truttel \& Wurz, 1844.

${ }^{20}$ Charles G.Leland, Fusang or the Discovery of America by Chinese Buddhist Priests in the Fifth Century, Londres, Trübner, 1875.

${ }^{21}$ Ed.P. Vinning, An inglorious Columbus, or evidence that Hwinshin and a party of buddhist monks of Afghanistan discovered America in the fifth century, New York, Appleton, 1885.

22 V. Orti Belmonte, Fué América conocida por los asiáticos antes de su descubrimiento por Colón? In: Boletín R.Artes, vol.VII, Córdoba 1928, p. 252.

${ }^{23}$ Julius Heinrich Klaproth, in: Nouvelles Annales des Voyages, tome XXI, $2^{\mathrm{e}}$ série, 1831.

24 Vivien de Saint Martin, Histoire de la Géographie et des découvertes géographiques, Paris 1875.

25 E. V.Bretschneider, Über das Land Fu-Sang, Yokohama 1876.

${ }^{26}$ Gustave Schlegel, Fou-Sang Kouo. Le Pays de Fou-sang (Extrait du T'oungPao, vol. III, n $^{\circ}$ 2). Leide, Brill, 1892.

27 Nous devons une partie de cette bibliographie à l'amabilité du Dr. Omar Ortiz Troncoso de l'Université d'Amsterdam. 
Henry Hammer, a German living at Florence, has drawn the coasts and rivers of South America on a world map of 1489 in the manuscript "Insularium Illustratum". Because the continent is situated on the east margin, it seems that it has been discovered from the Pacific Ocean. The author discusses this hypothesis. Perhaps the Egyptians knew Peru at 3000-1000 B.C., perhaps the Phoenicians after $1000 \mathrm{~B}$. C. (There have been found Phoenician rock inscriptions in the region of the Amazonas river.) It may be that the Chinese knew South America in the 5 th century A.D. or even some hundred years before. The decision depends on further historical or archaeological discoveries.

Prof. Dr. Pablo J. Gallez

Calle Peru 339

Bahia Blanca

Argentinien 